\title{
Post-Hoc Analyses of the Effects of Baseline Sleep Quality on SHP465 Mixed Amphetamine Salts Extended-Release Treatment Response in Adults with Attention-Deficit/Hyperactivity Disorder
}

\author{
Craig B. H. Surman ${ }^{1,2}$ (D) Brigitte Robertson ${ }^{3} \cdot$ Jie Chen $^{3} \cdot$ Samuele Cortese $^{4,5}$
}

Published online: 21 June 2019

(c) The Author(s) 2019

\begin{abstract}
Objective Sleep problems are common in adults with attention-deficit/hyperactivity disorder (ADHD). The presence of sleep problems at the time of presentation for ADHD treatment could impact the level of improvement in ADHD symptoms or executive function occurring with ADHD pharmacotherapy. Therefore, we examined the influence of baseline sleep quality on the effects of SHP465 mixed amphetamine salts (MAS) extended-release.

Methods Adults (18-55 years) with Diagnostic and Statistical Manual of Mental Disorders, Fourth Edition, Text Revisiondefined ADHD and baseline ADHD Rating Scale IV (ADHD-RS-IV) total scores $\geq 24$ were randomized to once-daily SHP465 MAS (12.5-75 mg) or placebo in a 7-week, double-blind, dose-optimization study. Post-hoc analyses evaluated SHP465 MAS treatment effects on ADHD symptoms, using the ADHD-RS-IV, and executive function, using the Brown Attention-Deficit Disorder Scale (BADDS), based on baseline sleep quality as defined by Pittsburgh Sleep Quality Index (PSQI) scores [sleep quality impaired (PSQI total score $>5$; PSQI component scores 2 or 3) versus not impaired (PSQI total score $\leq 5$; PSQI component scores 0 or 1)]. Analyses were conducted in the intent-to-treat population.

Results Of 280 enrolled participants, 272 were randomized (placebo, $n=135$; SHP465 MAS, $n=137$ ). The intent-totreat population consisted of 268 participants (placebo, $n=132$; SHP465 MAS, $n=136$ ), and 170 participants (placebo, $n=76$; SHP465 MAS, $n=94$ ) completed the study. Treatment differences nominally favored SHP465 MAS over placebo in both sleep impairment groups regarding ADHD-RS-IV total score changes (all nominal $p<0.05$ ), except for those with impairment defined by sleep efficiency $(p=0.2696)$, and regarding BADDS total score changes (all nominal $p<0.05)$, except for those with impairment defined by sleep duration $(p=0.1332)$ and sleep efficiency $(p=0.8226)$. There were no statistically significant differences in SHP465 MAS treatment effects between sleep impairment groups. Conclusions Improvements in ADHD symptoms and executive function occurred with dose-optimized SHP465 MAS, regardless of baseline impairment in some aspects of sleep in adults with ADHD, with no significant differences observed as a function of sleep impairment.
\end{abstract}

Clinical Trials Registration ClinicalTrials.gov identifier-NCT00150579.

Electronic supplementary material The online version of this article (https://doi.org/10.1007/s40263-019-00645-z) contains supplementary material, which is available to authorized users.

Craig B. H. Surman

csurman@partners.org

Extended author information available on the last page of the article

\section{Key Points}

Regardless of baseline sleep quality impairment, SHP465 MAS treatment was associated with nominal improvement in ADHD symptoms compared with placebo in adults diagnosed with ADHD.

Regardless of baseline sleep quality impairment, SHP465 MAS treatment was associated with nominal improvement in executive function compared with placebo in adults diagnosed with ADHD. 


\section{Introduction}

Attention-deficit/hyperactivity disorder (ADHD) is estimated to impact $4.4 \%$ of the US adult population [1] and $2.8 \%$ of adults worldwide; prevalence estimates range from as low as $0.6 \%$ in Romania and Iraq to as high as $7.3 \%$ in France [2]. Individuals diagnosed with ADHD may present with sleep problems [3-5]. Although these sleep problems may represent symptoms of comorbid mood or anxiety in some individuals, analyses correcting for such contributions from a community sample [4] and a longitudinal twin study [6] suggest ADHD is associated with sleep problems independent of psychiatric comorbidities.

It has been reported that at least some individuals with ADHD may present with hypo-arousal [7], which mimics ADHD-like symptoms, that might or might not improve with stimulant treatment. Alterations in arousal could also influence sleep quality and result in ADHD-like symptoms that might or might not improve with stimulant treatment. Sleep problems could also create a greater functional burden for individuals with ADHD. For example, impaired cognition has been shown to be associated with insomnia and sleep deprivation in several studies [8-11]. Individuals with primary insomnia exhibited worse executive function than 'good sleep' controls on an attention network test in the absence of significant differences in alertness, orientation, or reaction time [8]. In a study of individuals seeking treatment for insomnia, objectively measured full-scale intelligence quotient, verbal comprehension, memory, and processing speed were impaired relative to participants with insomnia not seeking treatment [9]. Lastly, sleep deprivation negatively impacts memory and mathematical processing in healthy adults $[10,11]$.

Theoretically, sleep problems at the time of presentation for ADHD treatment could impact the level of improvement in ADHD symptoms or executive function occurring with ADHD pharmacotherapy. Although how baseline sleep quality impacts treatment response to ADHD pharmacotherapy has not been evaluated to the best of our knowledge, such information could help clinicians optimize care for adults with ADHD and comorbid sleep problems.

The current post-hoc analyses use the Pittsburgh Sleep Quality Index (PSQI) to examine how baseline sleep quality impacts the effects of SHP465 mixed amphetamine salts (MAS) extended-release-a once-daily, single-entity MAS product for oral administration approved in the US for treating ADHD in patients $\geq 13$ years old [12]—on ADHD symptoms, measured with the ADHD Rating Scale version IV (ADHD-RS-IV), and self-reported executive function, measured with the Brown Attention-Deficit Disorder Scale (BADDS). The PSQI, a self-report questionnaire that assesses subjective sleep quality, has demonstrated internal consistency and construct validity [13] and has been used to assess sleep quality changes [14-16] in multiple clinical populations. In this short-term study of SHP465 MAS, the PSQI was included as a safety/tolerability endpoint and decreases in PSQI global score from baseline to the end of the study for SHP465 MAS were comparable with placebo [17]. However, the influence of baseline sleep quality on response to SHP465 MAS has not been examined.

Given the associations between arousal, ADHD, and sleep, it was hypothesized that baseline sleep quality might alter the magnitude of SHP465 MAS treatment effects on ADHD symptom reduction and/or improvement in executive function. It was hypothesized that SHP465 MAS treatment would be associated with greater improvement in ADHD symptoms and related executive function challenge in individuals with sleep problems due to the potential added benefit of stimulant treatment on hypoarousal. However, the possibility that sleep problems increase patient burden and make treatment less effective was also considered.

\section{Methods}

\subsection{Study Design and Treatment}

Detailed descriptions of the study design, participants, and endpoints have been reported [17]. This phase III, randomized, double-blind, placebo-controlled, parallel-group, dose-optimization study was conducted in the US (ClinicalTrials.gov: NCT00150579) [17]. All study procedures were conducted in accordance with ethical standards of the 18th World Medical Assembly and amendments of the 29th, 35th, 41st, and 48th World Medical Assemblies. The study was approved by either a central institutional review board (IRB) or local IRBs. Participants were required to provide written informed consent before study procedures were conducted.

The study included four phases (Fig. 1): screening ( 2 weeks), washout and baseline (7-28 days), double-blind treatment ( 7 weeks), and follow-up (4 weeks). During double-blind treatment, participants were randomized 1:1 to placebo or once-daily dose-optimized SHP465 MAS. Treatment started at $12.5 \mathrm{mg}$ SHP465 MAS. The dosage was increased weekly to 25,50 , and $75 \mathrm{mg}$ until an optimal dose (a dose associated with a $\geq 30 \%$ decrease in baseline ADHD-RSIV score and acceptable tolerability) was attained. Downtitration was allowed after 1 week at $50 \mathrm{mg}$ (to $37.5 \mathrm{mg}$ ) or $75 \mathrm{mg}$ (to $62.5 \mathrm{mg}$ ) SHP465 MAS. Once an optimal dosage was attained, it was maintained until the end of the study. Randomization was conducted using a centralized, interactive voice response system, with treatments assigned by a block-randomization schedule. 


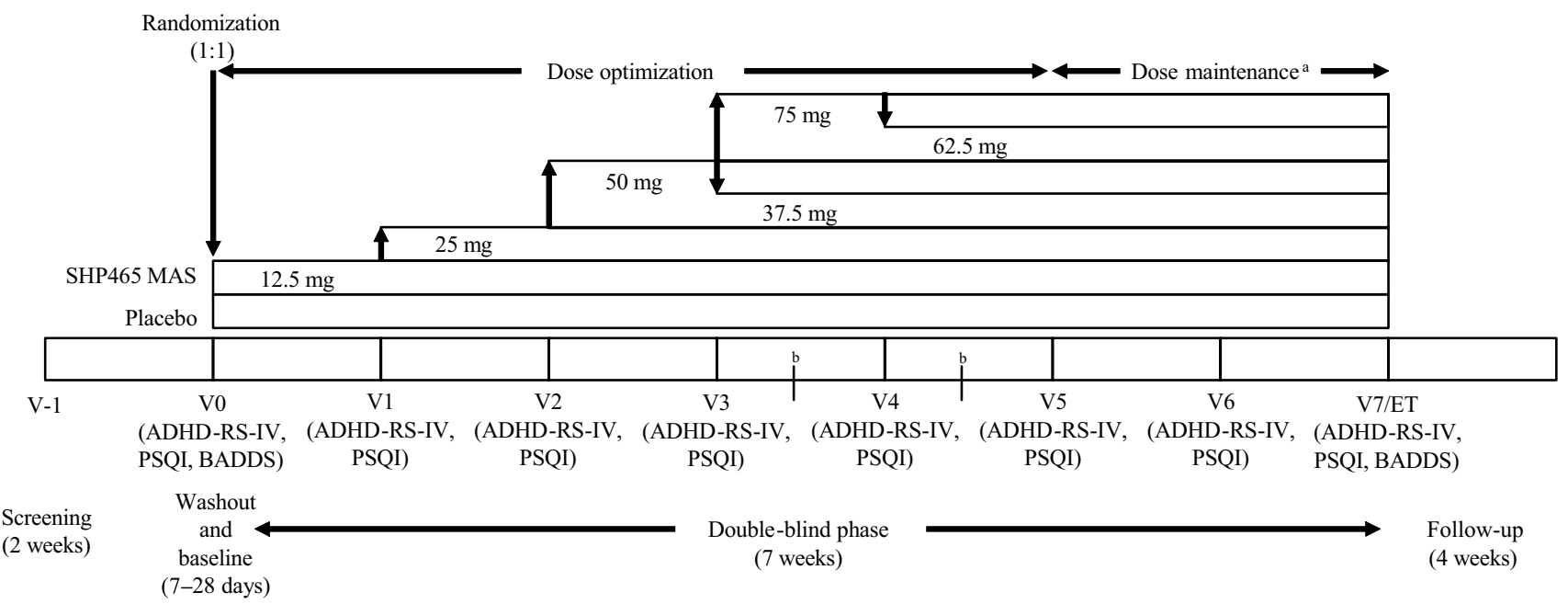

Fig. 1 Study design summary and timing of study assessments. $A D H D-R S-I V$ ADHD Rating Scale version IV, BADDS Brown Attention-Deficit Disorder Scale, ET early termination, MAS mixed amphetamine salts, PSQI Pittsburgh Sleep Quality Index, $V$ visit.

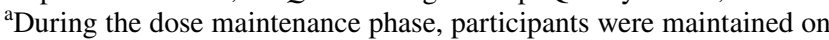

\subsection{Participants}

The study included adult men and nonpregnant/nonlactating women (18-55 years) with a confirmed ADHD diagnosis based on Diagnostic and Statistical Manual of Mental Disorders, Fourth Edition, Text Revision (DSM-IV-TR) criteria, baseline ADHD-RS-IV scores $\geq 24$, and satisfactory medical assessments with no clinically significant abnormalities based on medical history, physical examinations, and clinical and laboratory evaluations.

Individuals were excluded if they had a psychiatric comorbidity controlled with a prohibited medication or uncontrolled and associated with significant symptoms that contraindicated SHP465 MAS use or could confound assessments. Prohibited medications included anticonvulsants, antipsychotics, anxiolytics, benzodiazepines, clonidine or guanfacine, herbal preparations with psychoactive properties, investigational medications, monoamine oxidase inhibitors, psychostimulants, selective serotonin reuptake inhibitors, selective norepinephrine reuptake inhibitors, sedating antihistamines, sedative hypnotics, and tricyclic antidepressants. Additional exclusion criteria included having an illness or unstable medical condition that could confound study results, increase participant risk, or lead to difficulty complying with the protocol; cardiac structural anomalies; electrocardiogram or laboratory anomalies at screening or baseline; a history of hypertension; resting systolic blood pressure $>139 \mathrm{mmHg}$ or resting diastolic blood pressure $>89 \mathrm{mmHg}$; a history of intellectual disability or severe learning disability, seizure disorder other than infantile febrile seizures, a tic disorder, current diagnosis and/ the optimized dose established during the dose-optimization phases. ${ }^{\mathrm{b}}$ Midweek visits were required if participants were titrated to $75 \mathrm{mg}$ SHP465 MAS at visit 3 and if participants were downtitrated to $62.5 \mathrm{mg}$ MAS SHP465 at visit 4

or family history of Tourette syndrome; a history (within 6 months before screening) of drug dependence or substance abuse disorder according to DSM-IV-TR criteria, excluding nicotine; being underweight (body mass index $<18.5 \mathrm{~kg}$ / $\mathrm{m}^{2}$ ) or morbidly obese; using psychoactive medications (prescription or over-the-counter) requiring a washout period of $>28$ days; using investigational medications within 30 days of screening; and a documented allergy to, intolerance of, or history of nonresponsivity to methylphenidate or amphetamines.

\subsection{Measures}

The prespecified primary efficacy measure was the clinicianadministered ADHD-RS-IV. The 18-item ADHD-RS-IV, which includes two 9-item subscales (hyperactivity/impulsivity; inattentiveness), measures current ADHD symptoms [18]. Items are scored on 4-point scales [0 (no symptoms) to 3 (severe symptoms)], with total scores ranging from 0 to 54. In the current study, ADHD-RS-IV assessments were conducted at baseline and all study visits (Fig. 1).

The self-reported BADDS was a secondary efficacy measure. The BADDS consists of 40 items scored on 4-point scales $(0=$ never; $1=$ once a week or less; $2=$ twice a week; $3=$ almost daily) $[19,20]$. Items are grouped into five clusters (organizing and activating to work, sustaining attention and concentration, sustaining energy and effort, managing affective interference, and using working memory and accessing recall) of conceptually related ADHD symptoms [19]. BADDS total score ranges from 0 to 120, with scores for the 9-item organizing and activating to work, sustaining 
Table 1 PSQI component scoring

\begin{tabular}{|c|c|c|}
\hline PSQI score & Items and scoring $^{\mathrm{a}}$ & Component scoring \\
\hline Global & Sum of the individual component scores & Not applicable \\
\hline Sleep latency & $\begin{array}{l}\text { Sum of the individual responses to } \\
\text { Item } 2 \text { (how long to fall asleep): } 0 \text { ( } \leq 15 \mathrm{~min}), 1 \text { (16-30 min), } 2 \text { ( } 31-60 \mathrm{~min}), 3 \\
\text { ( }>60 \mathrm{~min}) \\
\text { Item } 5 \mathrm{a} \text { (cannot get to sleep within } 30 \mathrm{~min}): 0 \text { (not at all), } 1 \text { ( }<1 \text { time), } 2 \text { ( } 1 \text { or } 2 \\
\text { times), } 3 \text { ( } \geq 3 \text { times) }\end{array}$ & $\begin{array}{l}0 \text { (summed item range: } 0 \text { ) } \\
1 \text { (summed item range: } 1-2 \text { ) } \\
2 \text { (summed item range: } 3-4 \text { ) } \\
3 \text { (summed item range: } 5-6 \text { ) }\end{array}$ \\
\hline Sleep duration & Item 4 (hours of actual sleep) & $\begin{array}{l}0(>7 \mathrm{~h}) \\
1(6-7 \mathrm{~h}) \\
2(5-6 \mathrm{~h}) \\
3(<5 \mathrm{~h})\end{array}$ \\
\hline Sleep efficiency & $\begin{array}{l}\text { Efficiency calculation: (hours slept)/(waking time - bedtime hours in bed) } \times 100 \text { using } \\
\text { Item } 1 \text { (usual bedtime) } \\
\text { Item } 3 \text { (usual waking time) } \\
\text { Item } 4 \text { (hours slept) }\end{array}$ & $\begin{array}{l}0(>85 \%) \\
1(75-84 \%) \\
2(65-74 \%) \\
3(<65 \%)\end{array}$ \\
\hline Sleep disturbance & $\begin{array}{l}\text { Sum of the individual responses to } \\
\text { Items } 5 \mathrm{a} \text { (cannot get to sleep within } 30 \mathrm{~min}), 5 \mathrm{~b} \text { (wake up in middle of night/early } \\
\text { morning), } 5 \mathrm{c} \text { (get up to use bathroom), } 5 \mathrm{~d} \text { (cannot breathe comfortably), } 5 \mathrm{e} \text { (cough/ } \\
\text { snore loudly), } 5 \mathrm{f} \text { (feel too cold), } 5 \mathrm{~g} \text { (feel too hot), } 5 \mathrm{~h} \text { (have bad dreams), } 5 \mathrm{i} \text { (have } \\
\text { pain), } 5 \mathrm{j} \text { (other) } \\
\text { All scored as } 0 \text { (not at all), } 1 \text { ( }>1 \text { time), } 2 \text { ( } 1 \text { or } 2 \text { times), } 3 \text { ( } \geq 3 \text { times) }\end{array}$ & $\begin{array}{l}0 \text { (summed item range: } 0 \text { ) } \\
1 \text { (summed item range: } 1-9) \\
2 \text { (summed item range: } 10-18 \text { ) } \\
3 \text { (summed item range: } 19-27 \text { ) }\end{array}$ \\
\hline Daytime dysfunction & $\begin{array}{l}\text { Sum of the individual responses to } \\
\text { Item } 7 \text { (trouble staying awake while driving, eating, or engaging in social activity) } \\
\text { Item } 8 \text { (trouble keeping enthusiasm to get things done) } \\
\text { Both scored as } 0 \text { (not at all), } 1 \text { ( }>1 \text { time), } 2 \text { ( } 1 \text { or } 2 \text { times), } 3 \text { ( } \geq 3 \text { times) }\end{array}$ & $\begin{array}{l}0 \text { (summed item range: } 0 \text { ) } \\
1 \text { (summed item range: } 1-2 \text { ) } \\
2 \text { (summed item range: } 3-4) \\
3 \text { (summed item range: } 5-6 \text { ) }\end{array}$ \\
\hline
\end{tabular}

PSQI Pittsburgh Sleep Quality Index

${ }^{a}$ Based on the prior week

attention and concentration, and sustaining energy and effort clusters ranging from 0 to 27 , scores for the 7-item managing affective interference cluster ranging from 0 to 21 , and scores for the 6-item using working memory and accessing recall cluster ranging from 0 to 18 [19]. In this study, BADDS assessments were conducted at baseline and week 7/early termination (ET) (Fig. 1).

Sleep was assessed at baseline and all study visits (Fig. 1) as a safety/tolerability assessment using a modified 18-item PSQI, which assessed sleep quality during the past week. The modified PSQI used in this study differed from the validated PSQI [21] in that the validated PSQI assesses sleep habits over the last 30 days and includes input from a bed partner or roommate. The self-rated PSQI groups items into seven components (sleep quality, sleep latency, sleep duration, sleep efficiency, sleep disturbances, use of sleep medication, daytime dysfunction), with each component score rated on a scale ranging from 0 to 3 (higher scores indicate more severe impairment) [21]. Component scores are used to generate a global score ranging from 0 to 21 . In these analyses, five PSQI component scores and global score (Table 1) were used to categorize baseline sleep as either impaired or not impaired. The 'use of sleep medication' and 'sleep quality' components were not assessed because these components were thought to reflect sleep problems that overlapped with other PSQI components.

\subsection{Data Presentation and Analysis}

The prespecified primary and secondary efficacy findings, as well as safety and tolerability findings, from this study have been reported [17]. The post-hoc analyses described in this report examined changes from baseline to study endpoint (the average of weeks 5-7 or last post-randomization assessment if data from weeks 5-7 were missing) for ADHD-RS-IV scores and from baseline to week 7/ET for BADDS scores as function of baseline sleep quality (not impaired versus impaired). Baseline PSQI total scores $\leq 5$ were defined as sleep not impaired, and scores $>5$ were defined as sleep impaired, as has previously been described [22]. To the best of our knowledge, no publications describe cutoff values for sleep quality impairment based on PSQI component scores. Therefore, for the analyses based on PSQI components, scores of 0 or 1 were defined as sleep not impaired and scores of 2 or 3 were defined as sleep impaired. This dichotomization is justified because component items scored at 2 or 3 reflect higher impairment than those scored at 0 or 1 . 
Statistical analyses were conducted in the intent-totreat (ITT) population (randomized participants receiving one or more study drug dose and having one or more post-baseline primary efficacy assessments and a baseline assessment). Changes from baseline to endpoint in ADHD-RS-IV scores were analyzed using mixed-effects models for repeated measures. Changes from baseline to week 7/ET in BADDS scores were analyzed using analysis of covariance. Baseline age, body mass index, lifetime insomnia and lifetime depression based on medical history form responses, and respective baseline outcomes were included in the models as covariates because these factors could contribute to sleep quality and fatigue. A sensitivity analysis was also conducted in which baseline lifetime insomnia was not included as a covariate in the analyses. For comparisons between sleep impairment groups, reported $p$ values are based on interaction terms (sleep impairment $\times$ treatment) for the end-of-treatment visit in the primary models using the same baseline covariates. All reported $p$ values are nominal (unadjusted) and presented for descriptive purposes. Adjustments for multiple comparisons were not applied because the study was not powered for such adjustments.

\section{Results}

\subsection{Disposition and Demographics}

A full description of participant disposition is published [17]. In brief, 280 participants were enrolled and 272 were randomized (placebo, $n=135$; SHP465 MAS, $n=137)$. The ITT population consisted of 268 participants (placebo, $n=132$; SHP465 MAS, $n=136$ ), and 170 completed the study (placebo, $n=76$; SHP465 MAS, $n=94)$.

Demographics and baseline clinical characteristics are summarized in Table 2. Most participants were men [136/268 (50.7\%)], White [227/268 (84.7\%)], and diagnosed as having a combined ADHD presentation [190/268 (70.9\%)]. The mean \pm standard deviation (SD) age at baseline was $36.6 \pm 10.16$ years.

Most study participants had not been treated with ADHD pharmacotherapy before participating (placebo, 100/132; SHP465 MAS, 103/135). Since this study was not designed to assess sleep quality, melatonin use was not prohibited. However, only two participants (one in each treatment group) reported using melatonin before the study and only one participant in the SHP465 MAS group used melatonin during the study.

\subsection{ADHD-RS-IV Score Changes}

\subsubsection{ADHD-RS-IV Total Score}

Mean \pm SD ADHD-RS-IV total scores decreased from baseline with placebo and SHP465 MAS in participants with and without baseline impaired sleep quality, as measured by PSQI global or component scores (Fig. 2a). Least squares (LS) mean [95\% confidence interval (CI)] treatment differences for ADHD-RS-IV total score changes from baseline to endpoint favored SHP465 MAS over placebo (all nominal $p<0.001$ ) in participants without impaired sleep, as defined by PSQI global score and all components, and favored SHP465 MAS over placebo (all nominal $p<0.05$ ) in participants with impaired sleep, as defined by PSQI global score and all components except sleep efficiency (Fig. 3a). There were no statistically significant differences in drug-specific (SHP465 MAS - placebo) ADHD-RS-IV total score changes from baseline between sleep impairment groups (all nominal $p>0.05$ ).

\subsubsection{ADHD-RS-IV Subscale Scores}

LS mean $(95 \% \mathrm{CI})$ treatment differences for changes from baseline to endpoint favored SHP465 MAS over placebo for both subscales (all nominal $p<0.01$ ) in participants without baseline sleep impairment, as defined by PSQI global score and all components, and favored SHP465 MAS over placebo for both subscales (all nominal $p<0.01)$ in participants with baseline sleep impairment, as defined by PSQI global score and all components except sleep efficiency on the hyperactivity/impulsivity and inattentiveness subscales and sleep duration on the hyperactivity/impulsivity subscale (Fig. 3b, c). There were no statistically significant differences in drug-specific changes from baseline in ADHD-RS-IV subscale scores between sleep impairment groups (all nominal $p>0.05)$.

\subsection{BADDS Score Changes}

\subsubsection{BADDS Total Score}

Mean \pm SD BADDS total scores decreased from baseline with placebo and SHP465 MAS in participants with and without impaired sleep quality, as measured by PSQI global score and component scores (Fig. 2b). LS mean (95\% CI) treatment differences for BADDS total score changes from baseline to week 7/ET favored SHP465 MAS over placebo (all nominal $p<0.001$ ) in participants without impaired sleep, as defined by PSQI global score and all components, and favored SHP465 MAS over placebo (nominal $p<0.01$ ) in participants with impaired 
Table 2 Participant demographics and baseline clinical characteristics, ITT population

\begin{tabular}{|c|c|c|}
\hline & Placebo $(n=132)$ & SHP465 MAS $(n=136)$ \\
\hline Mean \pm SD age (years) & $37.1 \pm 10.26$ & $36.1 \pm 10.08$ \\
\hline \multicolumn{3}{|l|}{ Sex, $n(\%)$} \\
\hline Male & $67(50.8)$ & $69(50.7)$ \\
\hline Female & $65(49.2)$ & $67(49.3)$ \\
\hline \multicolumn{3}{|l|}{ Race, $n(\%)$} \\
\hline White & $110(83.3)$ & $117(86.0)$ \\
\hline Black & $12(9.1)$ & $9(6.6)$ \\
\hline Asian & $3(2.3)$ & $4(2.9)$ \\
\hline Other & $7(5.3)$ & $6(4.4)$ \\
\hline Mean \pm SD weight $(l b)^{a}$ & $178.9 \pm 40.90$ & $180.8 \pm 43.49$ \\
\hline \multicolumn{3}{|l|}{$\mathrm{BMI}^{\mathrm{b}}\left(\mathrm{kg} / \mathrm{m}^{2}\right)$} \\
\hline Mean \pm SD & $27.4 \pm 5.30$ & $27.7 \pm 5.40$ \\
\hline Median & 26.6 & 26.3 \\
\hline Range & $18-43$ & $18-44$ \\
\hline \multicolumn{3}{|l|}{ ADHD subtype, $n(\%)$} \\
\hline Inattentive & $33(25.0)$ & $37(27.2)$ \\
\hline Hyperactive/impulsive & $4(3.0)$ & $4(2.9)$ \\
\hline Combined & $95(72.0)$ & $95(69.9)$ \\
\hline Mean \pm SD ADHD-RS-IV total score & $36.0 \pm 7.44$ & $35.7 \pm 7.49$ \\
\hline Mean \pm SD BADDS total score & $79.7 \pm 17.08$ & $77.7 \pm 18.89$ \\
\hline Mean \pm SD PSQI total score ${ }^{c}$ & $6.9 \pm 3.25$ & $6.8 \pm 2.82$ \\
\hline
\end{tabular}

$A D H D$ attention-deficit/hyperactivity disorder, $A D H D-R S-I V$ ADHD Rating Scale version IV, BADDS Brown Attention-Deficit Disorder Scale, $B M I$ body mass index, ITT intent-to-treat, MAS mixed amphetamine salts, PSQI Pittsburgh Sleep Quality Index, $S D$ standard deviation

${ }^{a}$ Based on $n=135$ for SHP465 MAS

${ }^{\mathrm{b}}$ Based on randomized safety population: placebo, $n=135$; SHP465 MAS, $n=136$

${ }^{\mathrm{c}}$ Based on randomized safety population: placebo, $n=134$; SHP465 MAS, $n=137$

sleep, as defined by PSQI global score across all components except for sleep efficiency and sleep duration (Fig. 4a). There were no statistically significant differences in the drug-specific changes from baseline in BADDS total score between sleep impairment groups (all nominal $p>0.05$ ).

\subsubsection{BADDS Cluster Scores}

LS mean $(95 \% \mathrm{CI})$ treatment differences for changes from baseline to week 7/ET favored SHP465 MAS over placebo for all BADDS clusters (all nominal $p<0.05$ ) in participants without baseline sleep impairment, as defined by PSQI global score and all components, and favored SHP465 MAS over placebo for all BADDS clusters (all nominal $p<0.05$ ) in participants with baseline sleep impairment when impairment was defined by PSQI global score and the days dysfunctional due to sleepiness and sleep disturbance PSQI components (Fig. 4b-f). Treatment differences also favored SHP465 MAS over placebo in participants with baseline sleep impairment on the organizing and activating to work (nominal $p=0.0069$ ), sustaining attention and concentration (nominal $p=0.0043$ ), and using working memory and accessing recall (nominal $p<0.001$ ) BADDS clusters when impairment was defined by the PSQI sleep latency component (Fig. 4b, c, f). There were no significant differences in the drug-specific change from baseline in BADDS cluster scores between sleep impairment groups (all nominal $p>0.05$ ).

\subsection{Sensitivity Analyses: Impact of Baseline Lifetime Insomnia}

At baseline, 17 of 267 participants (placebo, $n=9$; SHP465 MAS, $n=8$ ) reported lifetime insomnia. Lifetime insomnia at baseline was reported more frequently by participants without baseline sleep quality impairment as measured by the days dysfunctional due to sleepiness, sleep duration, and sleep efficiency PSQI components and in participants with sleep quality impairment at baseline as measured by the sleep latency PSQI component [see Supplemental Table 1 in the electronic supplementary material (ESM)]. The results 
(a) ADHD-RS-IV total score changes

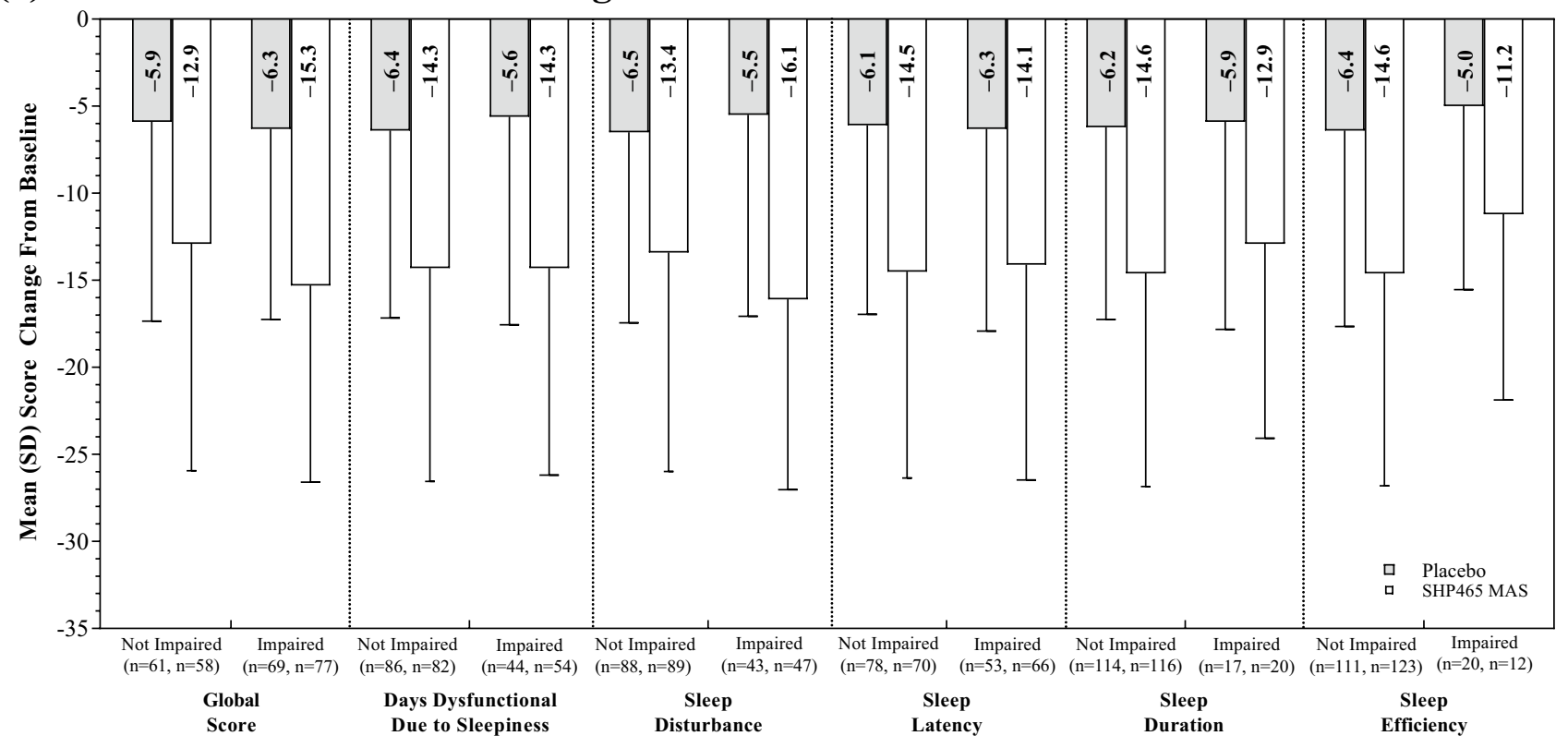

PSQI Component

(b) BADDS total score changes

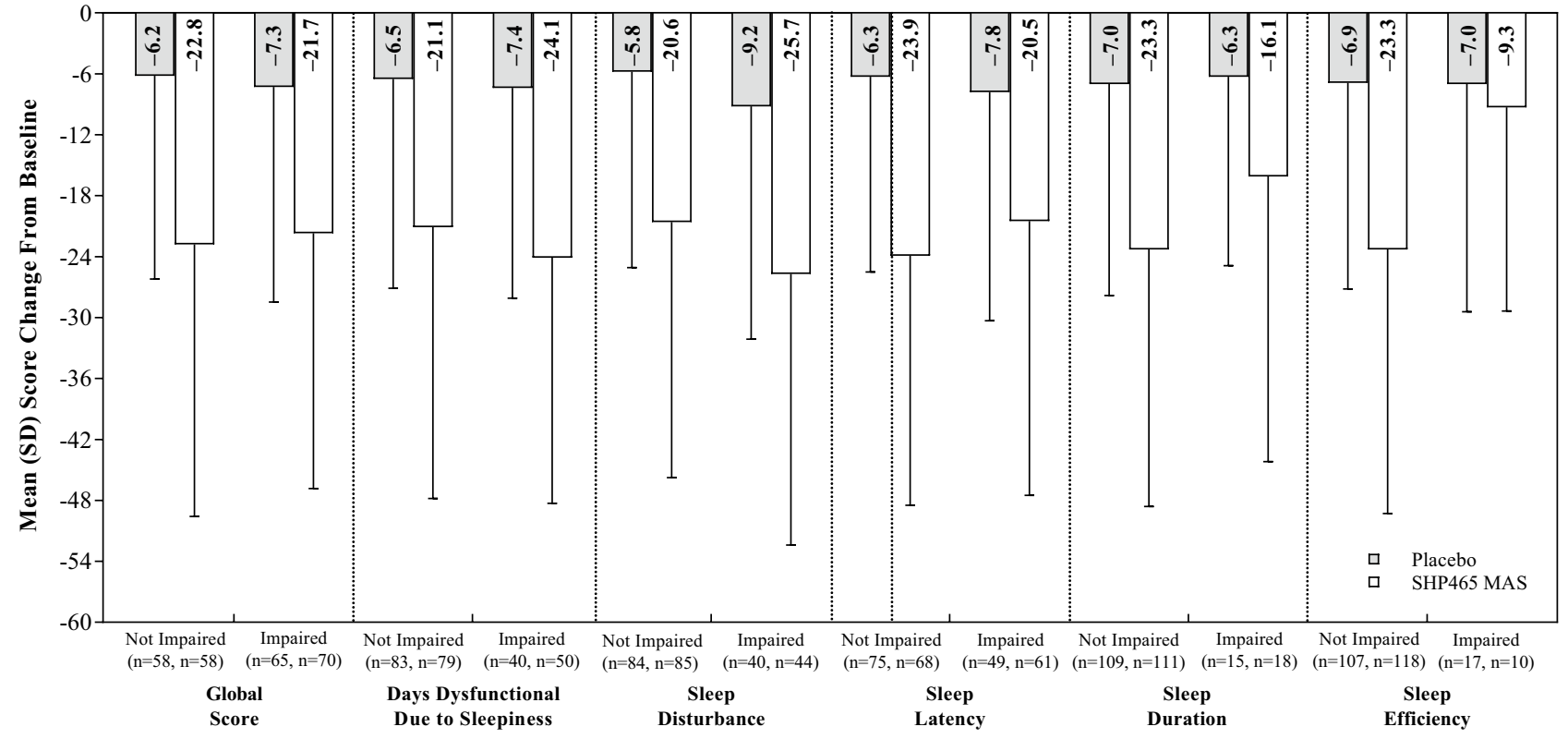

PSQI Component

Fig. 2 Change from baseline to endpoint in ADHD-RS-IV total score (a) and from baseline to week 7/ET in BADDS total score (b) by baseline sleep quality ${ }^{\mathrm{a}}$, ITT population. $A D H D-R S-I V$ Attention-Deficit/Hyperactivity Disorder Rating Scale, version IV, BADDS Brown Attention-Deficit Disorder Scale, ET early termination, ITT intent-to-

of sensitivity analyses that omitted baseline lifetime insomnia as a covariate were consistent with the primary analyses [see Supplemental Tables 2 and 3 (see ESM)]. treat, MAS mixed amphetamine salts, PSQI Pittsburgh Sleep Quality Index, $S D$ standard deviation. a Sleep quality not impaired (baseline PSQI global score $\leq 5$; baseline PSQI component score of 0 or 1 ), sleep quality impaired (baseline PSQI global score $>5$; baseline PSQI component score of 2 or 3 )

\section{Discussion}

This analysis examined the impact of baseline sleep quality impairment on SHP465 MAS treatment response in adults 


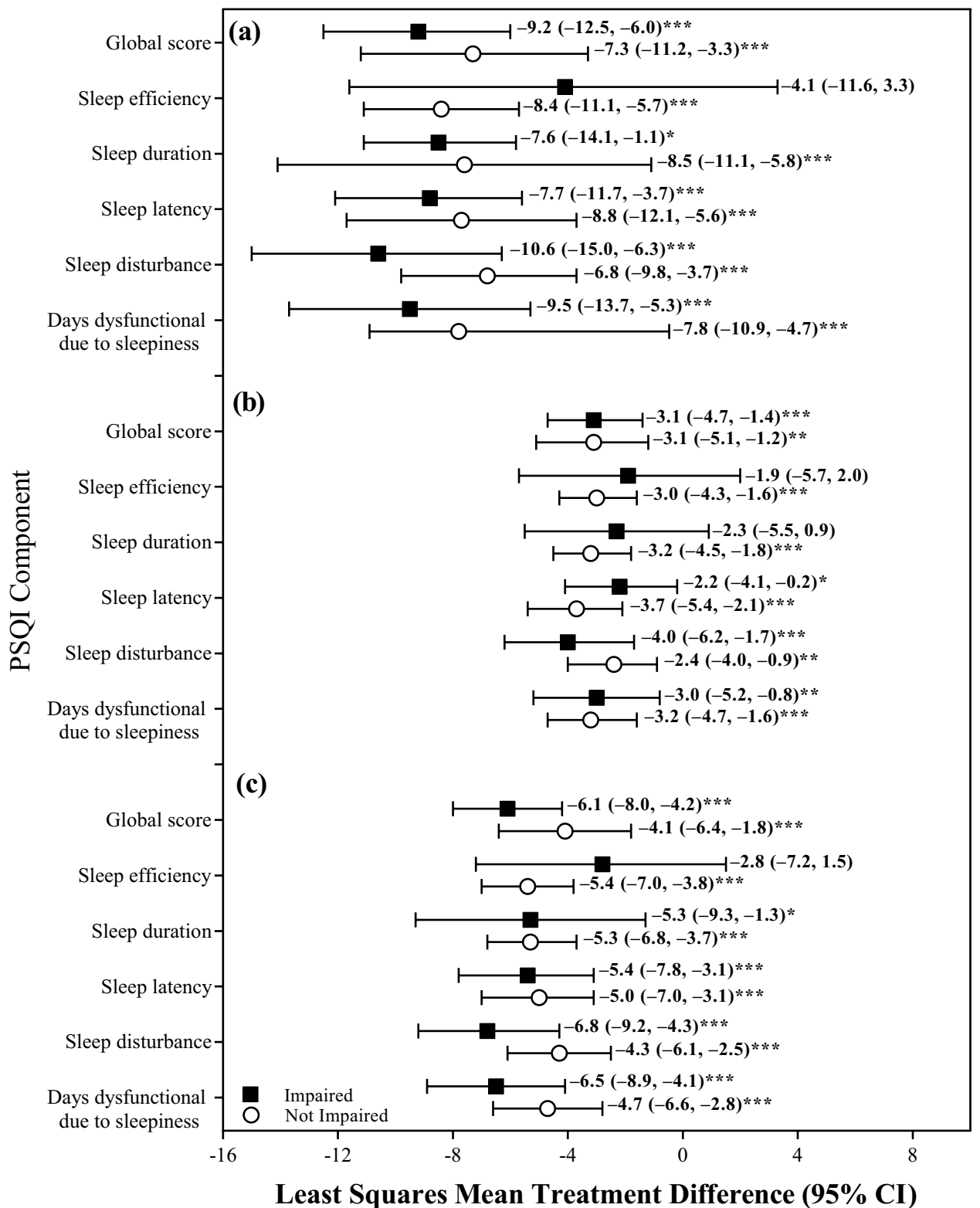

Fig. 3 Treatment differences (SHP465 MAS - placebo) in ADHDRS-IV total score change (a), hyperactivity/impulsivity subscale score change (b), and inattentiveness subscale score change (c) by baseline sleep quality ${ }^{\mathrm{a}}$, ITT population ${ }^{\mathrm{b}}$. ADHD-RS-IV Attention-Deficit/Hyperactivity Disorder Rating Scale, version IV, CI confidence interval, ITT intent-to-treat, MAS mixed amphetamine salts, PSQI

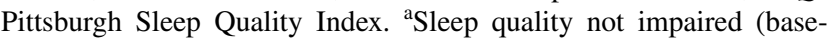
line PSQI global score $\leq 5$; baseline PSQI component score of 0 or 1 ), sleep quality impaired (baseline PSQI global score $>5$; baseline

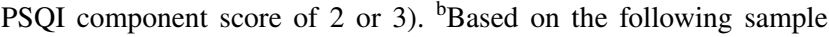
sizes: sleep quality not impaired [global score (placebo, 61; SHP465

with ADHD. Dose-optimized SHP465 MAS treatment resulted in significantly greater decreases in ADHD-RSIV and BADDS scores than placebo in a manner that was generally independent of baseline sleep quality. Although poor sleep quality measured by sleep duration and/or sleep
MAS, 58), sleep efficiency (placebo, 111; SHP465 MAS, 123), sleep duration (placebo, 114; SHP465 MAS, 116), sleep latency (placebo, 78; SHP465 MAS, 70), sleep disturbance (placebo, 88; SHP465 MAS, 89), days dysfunctional due to sleepiness (placebo, 86; SHP465 MAS, 82)]; sleep quality impaired [global score (placebo, 69; SHP465 MAS, 77), sleep efficiency (placebo, 20; SHP465 MAS, 12), sleep duration (placebo, 17; SHP465 MAS, 20), sleep latency (placebo, 53; SHP465 MAS, 66), sleep disturbance (placebo, 43; SHP465 MAS, 47), days dysfunctional due to sleepiness (placebo, 44; SHP465 MAS, 54)]. *Nominal $p<0.05$; **nominal $p \leq 0.01$; $* * *$ nominal $p \leq 0.001$ (SHP465 MAS versus placebo)

efficiency was associated with loss of drug-specific improvement, between-group comparisons indicated there were no differences on ADHD-RS-IV or BADDS scores as a function of baseline sleep quality. The observation that less separation from placebo was associated with poor sleep duration 
Fig. 4 Treatment differences (SHP465 MAS - placebo) in BADDS total (a) and cluster [organizing and activating to work (b), sustaining attention and concentration (c), sustaining energy and effort (d), managing affective interference (e), using working memory and accessing recall (f)] score changes by baseline sleep quality $^{\mathrm{a}}$, ITT population ${ }^{\mathrm{b}}$. $B A D D S$ Brown AttentionDeficit Disorder Scale, CI confidence interval, ITT intent-totreat, $M A S$ mixed amphetamine salts, PSQI Pittsburgh Sleep Quality Index. ${ }^{\text {aSleep quality }}$ not impaired (baseline PSQI global score $\leq 5$; baseline PSQI component score of 0 or 1 ), sleep quality impaired (baseline PSQI global score > 5; baseline PSQI component score of 2 or 3$)$. ${ }^{\mathrm{b}}$ Based on the following sample sizes: sleep quality not impaired [global score (placebo, 58; SHP465 MAS, 58 ), sleep efficiency (placebo, 107; SHP465 MAS, 118), sleep duration (placebo, 109; SHP465 MAS, 111), sleep latency (placebo, 75; SHP465 MAS, 68), sleep disturbance (placebo, 84; SHP465 MAS, 85), days dysfunctional due to sleepiness (placebo, 83; SHP465 MAS, 79)]; sleep quality impaired [global score (placebo, 65; SHP465 MAS, 70), sleep efficiency (placebo, 17; SHP465

MAS, 10), sleep duration (placebo, 15; SHP465 MAS, 18), sleep latency (placebo, 49; SHP465 MAS, 61), sleep disturbance (placebo, 40; SHP465 MAS, 44), days dysfunctional due to sleepiness (placebo, 40; SHP465 MAS, 50)]. *Nominal $p<0.05$; **nominal $p \leq 0.01$; $* * *$ nominal $p \leq 0.001$ (SHP465 MAS versus placebo)

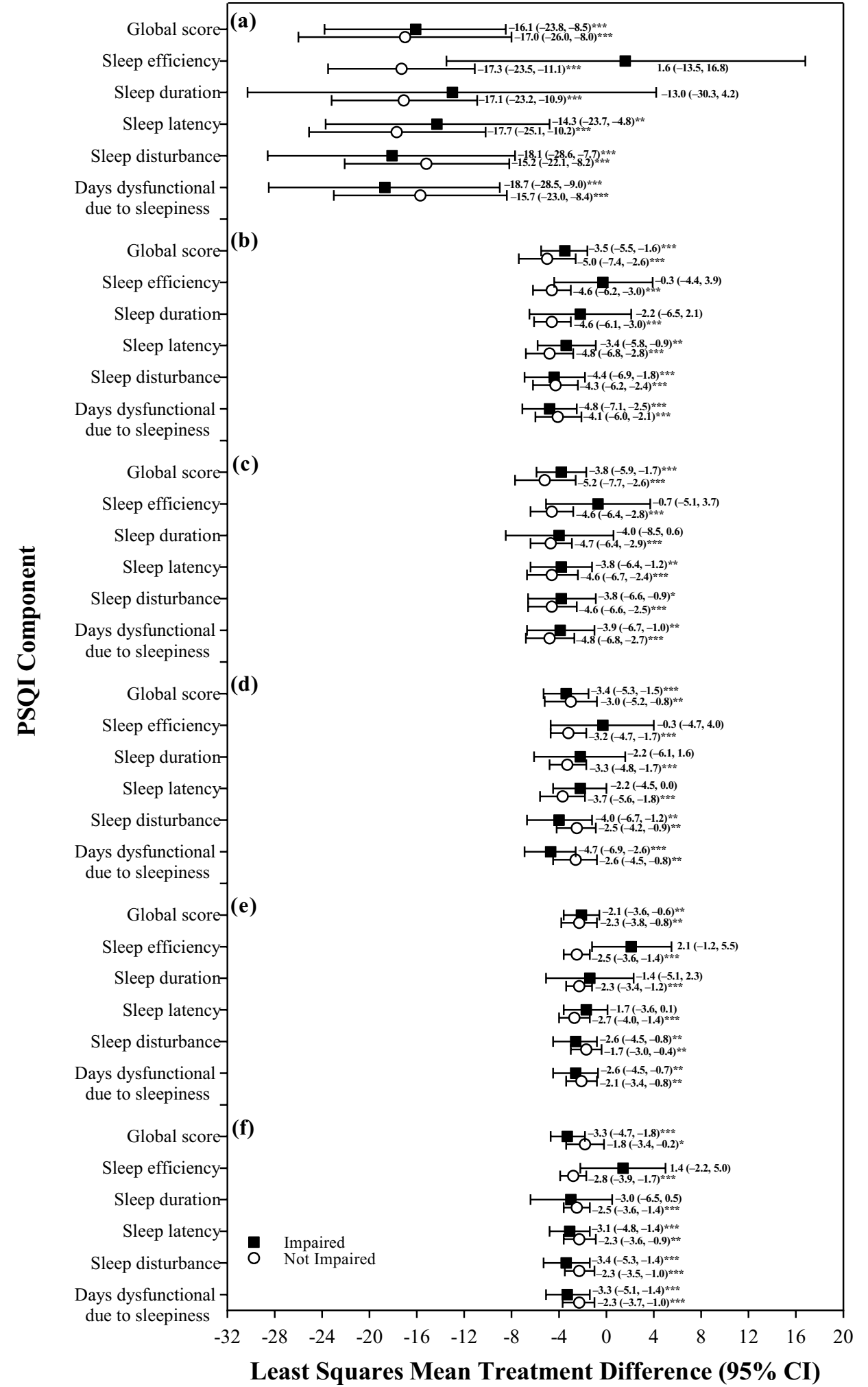

Least Squares Mean Treatment Difference (95\% CI) and/or poor sleep efficiency suggests additional examination might reveal that sleep problems attenuate the effects of ADHD pharmacotherapy. However, the lack of a drugspecific effect between sleep impairment groups indicates that the effects of SHP465 MAS are robust. This conclusion is further supported by the lack of drug-specific differences when baseline lifetime insomnia was not included as a covariate in the analyses. 
The lack of a robust effect of sleep quality impairment on SHP465 MAS treatment response was unexpected. In a conceptual model of interactions between ADHD and sleep, Hvolby posited that common overlapping neurobiologic mechanisms could give rise to both ADHD and sleep disturbances [23]. This supposition is supported by evidence that indicates monoaminergic systems are involved in the pathophysiology of ADHD [24] and the modulation of sleep and wakefulness [25]. Given that psychostimulants modulate monoaminergic systems [24], it was hypothesized that SHP465 MAS responses might be altered by baseline sleep impairment in a manner consistent with altered monoaminergic function. Given the lack of literature in this area, it is unclear if other indices or definitions of sleep impairment might moderate SHP465 MAS treatment responses in adults with ADHD. Studies in adults indicate the subjective sleep assessments based on the PSQI do not correlate with objective measures of sleep architecture assessed using polysomnography [26, 27], suggesting that these measures examine different aspects of sleep. Therefore, the possibility that categorization of sleep impairment based on objective measures would have revealed pronounced effects of sleep impairment on SHP465 MAS treatment response cannot be excluded.

From a clinical perspective, these data suggest sleep impairment in adults initiating SHP465 MAS for ADHD would not generally be expected to adversely influence the likelihood of a treatment response on the core symptoms of ADHD or executive function. However, given that stimulants can be associated with sleep disturbance in some, but not all, individuals $[23,28]$, it is important to monitor for sleep-related adverse effects of SHP465 MAS regardless of whether the individual being treated presents with sleep disturbances at treatment initiation. It should also be noted that a shift analysis of sleep quality was not conducted so it is not known if there were new-onset sleep problems associated with SHP465 MAS treatment.

The differential trends observed regarding SHP465 MAS treatment effects based on baseline sleep quality (i.e., a lack of nominal superiority of SHP465 MAS in those with baseline sleep quality impairment on some measures) suggest sleep quality may have reduced SHP465 MAS treatment responses. It is worth noting that between-group comparisons approached nominal significance for the BADDS clusters of organizing and activating to work (nominal $p=0.0898$ ), managing affective interference (nominal $p=0.0510$ ), and using working memory and accessing recall (nominal $p=0.0791$ ) when impairment was defined by the PSQI sleep efficiency component. In these instances, sample sizes were small in the sleep-impaired group (placebo, $n=17$; SHP465 MAS, $n=10$ ) and the effects of SHP465 MAS were either reduced or the opposite of what was observed in participants without impaired sleep. Additional analyses in larger populations are needed to more fully examine these relationships.
The study used for these analyses utilized dose optimization, which attempts to model clinical practice treatment paradigms. Therefore, it is hypothesized that these findings are generalizable to clinical practice. However, there are limitations to generalizing the findings obtained from a population of clinical trial participants, who are compensated for study participation, to individuals being treated in clinical practice. For example, although ADHD is often comorbid with other psychiatric disorders [29,30], this study excluded individuals with psychiatric comorbidities, which may have resulted in a healthier population with greater tolerance to stimulant effects. Other limitations should also be considered. First, adjustments for multiple comparisons were not applied because the study was not powered for such adjustments. As a result, all reported $p$ values are nominal and descriptive. Additional studies that are specifically powered for these assessments are needed to obtain more definitive results. Second, the sample size was small, particularly in some of the sleep impairment dichotomizations, and the data were highly variable. These issues limit the ability to interpret these data. Third, these findings are based on mean changes in the overall study population so the possibility that different response patterns occurred in individual participants cannot be excluded. Fourth, sleep impairment was defined using a subjective self-report that was included as a secondary safety endpoint. As subjective and objective sleep assessments may examine different aspects of sleep, it should be acknowledged that different results may have been observed if an objective sleep measure was used to define sleep impairment. Future analyses using objective sleep measures could provide additional insight into the effects of baseline sleep quality on SHP465 treatment response. Fifth, it is noteworthy that the PSQI was developed for evaluation of prior month experiences, but it was modified in this study to capture experiences during the prior week. This modification may have impacted the sensitivity or specificity of the sleep quality categorizations. Lastly, differences in ADHD-RS-IV and BADDS scores at baseline or study endpoint as a function of sleep quality impairment were not assessed. Such data could provide insight into why baseline sleep quality did not differentially affect SHP465 MAS treatment response.

Although this study was not designed to directly explore the hypothesis that arousal states are associated with ADHD, these findings lend support to the theory that sleep problems may be associated with states of hyperarousal or hypoarousal and ADHD symptoms. Because stimulants have wake-promoting effects that can interfere with sleep in some individuals, SHP465 MAS might be expected to result in lower levels of improvement in individuals with sleep problems who exhibit ADHD symptoms (e.g., hyperactivity associated with a state of hyperarousal). This phenomenon was potentially observed in individuals with ADHD reporting 
poor sleep quality, as defined by poor sleep efficiency and/ or sleep duration. These individuals experienced a relatively lower magnitude response to SHP465 MAS, particularly on the ADHD-RS-IV hyperactivity/impulsivity subscale. Alternatively, individuals presenting with ADHD who report sleep problems severe enough to cause sleep-related fatigue may be considered to be in a state of hypoarousal. These individuals could be somewhat resistant to the effects of SHP465 MAS, possibly due to a higher burden of brain-related challenges. Importantly, having individuals with hyperarousal or hypoarousal in the same study could influence the overall treatment effect observed for SHP465 MAS because individual responses and predictive factors might not be detected in an analysis of the overall population. However, the commonality in both arousal scenarios is a sleep problem that causes disturbances in arousal that present as ADHD and alter treatment response compared with individuals who present with a similar ADHD phenotype without associated sleep problems. Further studies specifically designed to evaluate the relationship between arousal and stimulant responsiveness are needed to better understand the association between arousal and ADHD. Future analyses examining the treatment effects over time and as a function of SHP465 MAS dose, as well as the relationship between study discontinuation and baseline sleep quality, could also provide additional insight into the impact of sleep quality on SHP465 MAS treatment effects.

\section{Conclusions}

To the best of our knowledge, this is the first analysis evaluating sleep impairment as a mediator of treatment outcome with ADHD pharmacotherapy. Our findings suggest that SHP465 MAS has favorable effects on the core symptoms of ADHD and on executive function in the presence of sleep problems at baseline. Some differential trends were observed as a function of how impaired sleep quality was defined, with impairment in sleep efficiency being associated with trends toward reduced magnitude of the SHP465 MAS treatment responses versus placebo. However, inferential analyses indicated there were no differences in the effects of SHP465 MAS on ADHD symptoms or executive function based on baseline sleep quality. Therefore, evidence supporting a role for sleep impairment mediating stimulant outcome was not found based on the current analyses. Further analyses that explore the moderating effects of sleep quality characteristics on ADHD symptoms and executive function using path analyses are needed to more thoroughly examine these relationships. If our results are confirmed by further studies, baseline sleep impairment should not be considered by practitioners to be a factor limiting treatment response to amphetamine-based stimulants in individuals with ADHD. Despite these findings, when treating patients with sleep impairment and ADHD, the evaluation of outcomes both pre- and post-treatment is recommended.

Acknowledgements Under the direction of the authors, Craig Slawecki, Ph.D., an employee of Complete Healthcare Communications, LLC (CHC; North Wales, PA, USA), a CHC group company, provided writing assistance for this manuscript. Shire Development LLC, Lexington, MA, USA, a member of the Takeda group of companies, provided funding to $\mathrm{CHC}$ for support in writing and editing this manuscript.

\section{Compliance with Ethical Standards}

Funding This clinical research was funded by Shire Development LLC, Lexington, MA, USA, a member of the Takeda group of companies. The open access fee for this manuscript was paid for by Shire Development LLC, Lexington, MA, USA, a member of the Takeda group of companies.

Conflict of interest C. B. H. Surman reports disclosures for speaking/ education for Arbor, McNeil, Janssen, Janssen-Ortho, Novartis, Shire, Reed/Massachusetts General Hospital Academy, Global Medical Education, and the North American Center for Continuing Medical Education (the latter three funded by multiple companies); research support for the Massachusetts General Hospital Adult ADHD Program (National Institutes of Health, Abbott, Cephalon, Hilda and Preston Davis Foundation, Eli Lilly, Magceutics, J \& J/McNeil, Merck, Magceutics/ Neurocentria, Nordic Naturals, Nestle/Pamlab, Pfizer, Organon, Shire, and Takeda); consulting for McNeil, NLS Pharma, Nutricia/Dannone, Purdue, Rhodes, Shire, Somaxon, Sunovion, Supernus, Takeda, Teva, and Vaya; and book royalties for Fast Minds-How to Thrive If You Have ADHD [or Think You Might]; ADHD in Adults-A Practical Guide to Evaluation and Management. B. Robertson and J. Chen are employees of Shire, a member of the Takeda group of companies, and hold Takeda stock. S. Cortese reports not receiving any honorarium for this work; he has received honoraria from the Association of Child and Adolescent Mental Health (ACAMH), a United Kingdom charity, for speaking about ADHD.

Ethical approval All study procedures were conducted in accordance with the ethical standards of the 18th World Medical Assembly and amendments of the 29th, 35th, 41st, and 48th World Medical Assemblies. The study was approved by either a central institutional review board (IRB) or local IRBs.

Informed consent Participants were required to provide written informed consent before study procedures were conducted.

Open Access This article is distributed under the terms of the Creative Commons Attribution-NonCommercial 4.0 International License (http://creativecommons.org/licenses/by-nc/4.0/), which permits any noncommercial use, distribution, and reproduction in any medium, provided you give appropriate credit to the original author(s) and the source, provide a link to the Creative Commons license, and indicate if changes were made.

\section{References}

1. Kessler RC, Adler L, Barkley R, et al. The prevalence and correlates of adult ADHD in the United States: results from the 
National Comorbidity Survey Replication. Am J Psychiatry. 2006;163:716-23.

2. Fayyad J, Sampson NA, Hwang I, et al. The descriptive epidemiology of DSM-IV adult ADHD in the World Health Organization World Mental Health Surveys. Atten Defic Hyperact Disord. 2017;9:47-65.

3. Diaz-Roman A, Mitchell R, Cortese S. Sleep in adults with ADHD: systematic review and meta-analysis of subjective and objective studies. Neurosci Biobehav Rev. 2018;89:61-71.

4. Surman CB, Adamson JJ, Petty C, et al. Association between attention-deficit/hyperactivity disorder and sleep impairment in adulthood: evidence from a large controlled study. J Clin Psychiatry. 2009;70:1523-9.

5. Bjorvatn B, Brevik EJ, Lundervold AJ, et al. Adults with attention deficit hyperactivity disorder report high symptom levels of troubled sleep, restless legs, and cataplexy. Front Psychol. 2017;8:1621.

6. Gregory AM, Agnew-Blais JC, Matthews T, et al. ADHD and sleep quality: longitudinal analyses from childhood to early adulthood in a twin cohort. J Clin Child Adolesc Psychol. 2017;46:284-94.

7. James SN, Cheung CHM, Rijsdijk F, et al. Modifiable arousal in attention-deficit/hyperactivity disorder and its etiological association with fluctuating reaction times. Biol Psychiatry Cogn Neurosci Neuroimaging. 2016;1:539-47.

8. Liu H, Wang D, Li Y, et al. Examination of daytime sleepiness and cognitive performance testing in patients with primary insomnia. PLoS One. 2014;9:e100965.

9. Goldman-Mellor S, Caspi A, Gregory AM, et al. Is insomnia associated with deficits in neuropsychological functioning? Evidence from a population-based study. Sleep. 2015;38:623-31.

10. Xu J, Zhu Y, Fu C, et al. Frontal metabolic activity contributes to individual differences in vulnerability toward total sleep deprivation-induced changes in cognitive function. J Sleep Res. 2016;25:169-80

11. Lo JC, Chong PL, Ganesan S, et al. Sleep deprivation increases formation of false memory. J Sleep Res. 2016;25:673-82.

12. Mydayis ${ }^{\circledR}$ extended-release capsules (mixed salts of a single-entity amphetamine product). Full Prescribing Information, Shire US Inc., Lexington; 2017.

13. Carpenter JS, Andrykowski MA. Psychometric evaluation of the Pittsburgh Sleep Quality Index. J Psychosom Res. 1998;45:5-13.

14. Nierenberg AA, Adler LA, Peselow E, et al. Trazodone for antidepressant-associated insomnia. Am J Psychiatry. 1994;151:1069-72.

15. Tremmas I, Petsatodis G, Potoupnis M, et al. Monitoring changes in quality of life in patients with lung cancer under treatment with chemotherapy and co administration of zoledronic acid by using specialized questionnaires. J Cancer. 2018;9:1731-6.

16. Tsunoda T, Yamada M, Akiyama T, et al. The effects of ramelteon on glucose metabolism and sleep quality in type 2 diabetic patients with insomnia: a pilot prospective randomized controlled trial. J Clin Med Res. 2016;8:878-87.

17. Spencer TJ, Adler LA, Weisler RH, et al. Triple-bead mixed amphetamine salts (SPD465), a novel, enhanced extended-release amphetamine formulation for the treatment of adults with ADHD: a randomized, double-blind, multicenter, placebo-controlled study. J Clin Psychiatry. 2008;69:1437-48.

18. Dupaul G, Power T, Anastopoulos A, et al. ADHD Rating ScaleIV: checklists, norms, and clinical interpretation. New York: Guilford Press; 1998.

19. Brown TE. Brown attention-deficit hyperactivity disorder scales for adolescents and adults. San Antonio: The Psychological Corporation; 1996.

20. Brown TE, Landgraf JM. Improvements in executive function correlate with enhanced performance and functioning and health-related quality of life: evidence from 2 large, double-blind, randomized, placebo-controlled trials in ADHD. Postgrad Med. 2010;122:42-51.

21. Buysse DJ, Reynolds CF 3rd, Monk TH, et al. The Pittsburgh Sleep Quality Index: a new instrument for psychiatric practice and research. Psychiatry Res. 1989;28:193-213.

22. Surman $\mathrm{CB}$, Roth $\mathrm{T}$. Impact of stimulant pharmacotherapy on sleep quality: post hoc analyses of 2 large, double-blind, randomized, placebo-controlled trials. J Clin Psychiatry. 2011;72:903-8.

23. Hvolby A. Associations of sleep disturbance with ADHD: implications for treatment. Atten Defic Hyperact Disord. 2015;7:1-18.

24. Faraone SV. The pharmacology of amphetamine and methylphenidate: relevance to the neurobiology of attention-deficit/hyperactivity disorder and other psychiatric comorbidities. Neurosci Biobehav Rev. 2018;87:255-70.

25. Schwartz MD, Kilduff TS. The neurobiology of sleep and wakefulness. Psychiatr Clin N Am. 2015;38:615-44.

26. Buysse DJ, Hall ML, Strollo PJ, et al. Relationships between the Pittsburgh Sleep Quality Index (PSQI), Epworth Sleepiness Scale (ESS), and clinical/polysomnographic measures in a community sample. J Clin Sleep Med. 2008;4:563-71.

27. Landry GJ, Best JR, Liu-Ambrose T. Measuring sleep quality in older adults: a comparison using subjective and objective methods. Front Aging Neurosci. 2015;7:166.

28. Stein MA, Weiss M, Hlavaty L. ADHD treatments, sleep, and sleep problems: complex associations. Neurotherapeutics. 2012;9:509-17.

29. Kooij JJ, Huss M, Asherson P, et al. Distinguishing comorbidity and successful management of adult ADHD. J Atten Disord. 2012;16:3S-19S.

30. Mao AR, Findling RL. Comorbidities in adult attention-deficit/ hyperactivity disorder: a practical guide to diagnosis in primary care. Postgrad Med. 2014;126:42-51.

\section{Affiliations}

\section{Craig B. H. Surman ${ }^{1,2}\left(\mathbb{D} \cdot\right.$ Brigitte Robertson $^{3} \cdot$ Jie Chen $^{3} \cdot$ Samuele Cortese $e^{4,5}$}

1 Clinical and Research Program in Pediatric

Psychopharmacology and Adult ADHD, Massachusetts

General Hospital, Harvard Medical School, 55 Fruit Street, Boston, MA 02114, USA

2 Harvard Medical School, Boston, MA, USA

3 Shire, a member of the Takeda group of companies, Lexington, MA, USA
4 University of Southampton and Solent NHS Trust, Southampton, UK

5 New York University Child Study Center, New York, NY, USA 